\title{
Classifying chart cells for quadratic complexity context-free inference
}

\author{
Brian Roark and Kristy Hollingshead \\ Center for Spoken Language Understanding \\ Oregon Health \& Science University, Beaverton, Oregon, 97006 USA \\ \{roark, hollingk\}@eslu.ogi.edu
}

\begin{abstract}
In this paper, we consider classifying word positions by whether or not they can either start or end multi-word constituents. This provides a mechanism for "closing" chart cells during context-free inference, which is demonstrated to improve efficiency and accuracy when used to constrain the wellknown Charniak parser. Additionally, we present a method for "closing" a sufficient number of chart cells to ensure quadratic worst-case complexity of context-free inference. Empirical results show that this $O\left(n^{2}\right)$ bound can be achieved without impacting parsing accuracy.
\end{abstract}

\section{Introduction}

While there have been great advances in the statistical modeling of hierarchical syntactic structure in the past 15 years, exact inference with such models remains very costly, so that most rich syntactic modeling approaches involve heavy pruning, pipelining or both. Pipeline systems make use of simpler models with more efficient inference to reduce the search space of the full model. For example, the well-known Ratnaparkhi (1999) parser used a POS-tagger and a finite-state NP chunker as initial stages of a multi-stage Maximum Entropy parser. The Charniak (2000) parser uses a simple PCFG to prune the chart for a richer model; and Charniak and Johnson (2005) added a discriminatively trained reranker to the end of that pipeline.

Recent results making use of finite-state chunkers early in a syntactic parsing pipeline have shown both an efficiency (Glaysher and Moldovan, 2006) and an accuracy (Hollingshead and Roark, 2007) benefit to the use of such constraints in a parsing system. Glaysher and Moldovan (2006) demonstrated an efficiency gain by explicitly disallowing entries in chart cells that would result in constituents that cross chunk boundaries. Hollingshead and Roark (2007) demonstrated that high precision constraints on early stages of the Charniak and Johnson (2005) pipeline-in the form of base phrase constraints derived either from a chunker or from later stages of an earlier iteration of the

(C) 2008. Licensed under the Creative Commons Attribution-Noncommercial-Share Alike 3.0 Unported license (http://creativecommons.org/licenses/by-nc-sa/3.0/). Some rights reserved. same pipeline-achieved significant accuracy improvements, by moving the pipeline search away from unlikely areas of the search space. Both of these approaches (as with Ratnaparkhi earlier) achieve their improvements by ruling out parts of the search space for downstream processes, and the gain can either be realized in efficiency (same accuracy, less time) or accuracy (same time, greater accuracy). Parts of the search space are ruled out precisely when they are inconsistent with the generally reliable output of the chunker, i.e., the constraints are a by-product of chunking.

In this paper, we consider building classifiers that more directly address the problem of "closing" chart cells to entries, rather than extracting this information from taggers or chunkers built for a different purpose. We build two classifiers, which tag each word in the sequence with a binary class label. The first classifier decides if the word can begin a constituent of span greater than one word; the second classifier decides if the word can end a constituent of span greater than 1 . Given a chart cell $(i, j)$ with start word $w_{i}$ and end word $w_{j}$, where $j>i$, that cell can be "closed" to entries if the first classifier decides that $w_{i}$ cannot be the first word of a multi-word constituent or if the second classifier decides that $w_{j}$ cannot be the last word in a multiword constituent. In such a way, we can optimize classifiers specifically for the task of constraining chart parsers. Note that such classifier output would be relatively straightforward to incorporate into most existing context-free constituent parsers.

We demonstrate the baseline accuracies of such classifiers, and their impact when the constraints are placed on the Charniak and Johnson (2005) parsing pipeline. Various ways of using classifier output are investigated, including one method for guaranteeing quadratic complexity of the contextfree parser. A proof of the quadratic complexity is included, along with a detailed performance evaluation when constraining the Charniak parser to be worst-case quadratic.

\section{Background}

Dynamic programming for context-free inference generally makes use of a chart structure, as shown in Fig. 1. Each cell in the chart represents a possible constituent spanning a substring, which is identified by the indices of the first and last words of the substring. Thus, the cell identified with 


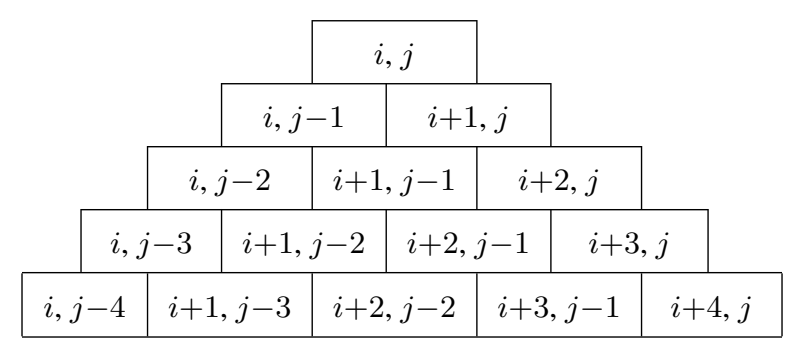

Figure 1: Fragment of a chart structure. Each cell is indexed with start and end word indices.

$i, j$ will contain possible constituents spanning the substring $w_{i} \ldots w_{j}$. Context-free inference has cubic complexity in the length of the string $n$, due to the $O\left(n^{2}\right)$ chart cells and $O(n)$ possible child configurations at each cell. For example, the CYK algorithm, which assumes a grammar in Chomsky Normal Form (hence exactly 2 non-terminal children for each constituent of span greater than 1), must consider the $O(n)$ possible midpoints for the two children of constituents at each cell.

In a parsing pipeline, some decisions about the hidden structure are made at an earlier stage. For example, base phrase chunking involves identifying a span as a base phrase of some category, often NP. A base phrase constituent has no children other than pre-terminal POS-tags, which all have a single terminal child, i.e., there is no internal structure in the base phrase involving nonPOS non-terminals. This has a number of implications for the context-free parser. First, there is no need to build internal structure within the identified base phrase constituent. Second, constituents which cross brackets with the base phrase cannot be part of the final tree structure. This second constraint on possible trees can be thought of as a constraint on chart cells, as pointed out in Glaysher and Moldovan (2006): no multi-word spanning constituent can begin at a word falling within a base-phrase chunk, other than the first word of that chunk. Similarly, no multi-word spanning constituent can end at a word falling within a base-phrase chunk, other than the last word of that chunk. These constraints rule out many possible structures that the full context-free parser would have to otherwise consider.

These start and end constraints can be extracted from the output of the chunker, but the chunker is not trained to optimize the accuracy (or the precision) of these particular constraints, rather typically to optimize chunking accuracy. Further, these constraints can apply even for words which fall outside of typical chunks. For example, in English, verbs and prepositions tend to occur before their arguments, hence are often unlikely to end constituents, despite not being inside a typically defined base phrase. If we can build a classifier specifically for this task (determining whether a

\begin{tabular}{|c|c|c|}
\hline Strings in corpus & 39832 & \\
\hline Word tokens in corpus & 950028 & \\
\hline Tokens neither first nor last in string & 870399 & \\
\hline Word tokens in $S_{1}$ & 439558 & $50.5 \%$ \\
\hline Word tokens in $E_{1}$ & 646855 & $74.3 \%$ \\
\hline
\end{tabular}

Table 1: Statistics on word classes from sections 2-21 of the Penn Wall St. Journal Treebank

word can start or end a multi-word constituent), we can more directly optimize the classifier for use within a pipeline.

\section{Starting and ending constituents}

To better understand the particular task that we propose, and its likely utility, we first look at the distribution of classes and our ability to build simple classifiers to predict these classes. First, let us introduce notation. Given a string of $n$ words $w_{1} \ldots w_{n}$, we will say that a word $w_{i}(1<i<n)$ is in the class $S_{>1}$ if there is a constituent spanning $w_{i} \ldots w_{j}$ for some $j>i$; and $w_{i} \in S_{1}$ otherwise. Similarly, we will say that a word $w_{j}(1<j<n)$ is in the class $E_{>1}$ if there is a constituent spanning $w_{i} \ldots w_{j}$ for some $i<j$; and $w_{j} \in E_{1}$ otherwise. These are two separate binary classification tasks.

Note that the first word $w_{1}$ and the last word $w_{n}$ are unambiguous in terms of whether they start or end constituents of length greater than 1 . The first word $w_{1}$ must start a constituent spanning the whole string, and the last word $w_{n}$ must end that same constituent. The first word $w_{1}$ cannot end a constituent of length greater than 1; similarly, the last word $w_{n}$ cannot start a constituent of length greater than 1. Hence our classifier evaluation omits those two word positions, leading to $n-2$ classifications for a string of length $n$.

Table 1 shows statistics from sections 2-21 of the Penn WSJ Treebank (Marcus et al., 1993). From the nearly 1 million words in approximately 40 thousand sentences, just over 870 thousand are neither the first nor the last word in the string, hence possible members of the sets $S_{1}$ or $E_{1}$, i.e., not beginning a multi-word constituent $\left(S_{1}\right)$ or not ending a multi-word constituent $\left(E_{1}\right)$. Of these, over half $(50.5 \%)$ do not begin multi-word constituents, and nearly three quarters $(74.3 \%)$ do not end multi-word constituents. This high latter percentage reflects English right-branching structure.

How well can we perform these binary classification tasks, using simple (linear complexity) classifiers? To investigate this question, we used sections 2-21 of the Penn WSJ Treebank as training data, section 00 as heldout, and section 24 as development. Word classes are straightforwardly extracted from the treebank trees, by measuring the span of constituents starting and ending at each word position. We trained log linear models with the perceptron algorithm (Collins, 2002) using fea- 


\begin{tabular}{|l|c|c|c|}
\hline \multirow{2}{*}{ Classification Task } & \multicolumn{3}{|c|}{ Markov order } \\
\cline { 2 - 4 } & 0 & 1 & 2 \\
\hline$S_{1}$ (no multi-word constituent start) & 96.7 & 96.9 & 96.9 \\
\hline$E_{1}$ (no multi-word constituent end) & 97.3 & 97.3 & 97.3 \\
\hline
\end{tabular}

Table 2: Classification accuracy on development set for binary classes $S_{1}$ and $E_{1}$, for various Markov orders.

tures similar to those used for NP chunking in Sha and Pereira (2003), including surrounding POStags (provided by a separately trained log linear POS-tagger) and surrounding words, up to 2 before and 2 after the current word position.

Table 2 presents classification accuracy on the development set for both of these classification tasks. We trained models with Markov order 0 (each word classified independently), order 1 (features with class pairs) and order 2 (features with class triples). This did not change performance for the $E_{1}$ classification, but Markov order 1 was slightly (but significantly) better than order 0 for $S_{1}$ classification. Hence, from this point forward, all classification will be Markov order 1 .

We can see from these results that simple classification approaches yield very high classification accuracy. The question now becomes, how can classifier output be used to constrain a context-free parser, and what is the impact on parser performance of using such a classifier in the pipeline.

\section{Closing chart cells}

Before moving on to an empirical investigation of constraining context-free parsers with the methods we propose, we first need to take a fairly detailed look at representations internal to these parsers. In particular, while we can rule out multi-word constituents with particular start and end positions, there may be intermediate or incomplete structures within the parser that should not be ruled out at these same start and end positions. Hence the notion of "closing" a chart cell is slightly more complicated than it may seem initially.

Consider the chart representation in Fig. 1. Suppose that $w_{i}$ is in class $S_{1}$ and $w_{j}$ is in class $E_{1}$, for $i<j$. We can "close" all cells $(i, k)$ such that $i<k$ and all cells $(l, j)$ such that $l<j$, based on the fact that multi-word constituents cannot begin with word $w_{i}$ and cannot end with $w_{j}$. A closed cell will not take complete entries, and, depending on the constraint used to close the cell, will have restrictions on incomplete entries. To make this more explicit, let us precisely define complete and incomplete entries.

Context-free inference using dynamic programming over a chart structure builds longer-span constituents by combining smaller span constituents, guided by rules in a context-free grammar. A context-free grammar $G=\left(V, T, S^{\dagger}, P\right)$ consists of: a set of non-terminal symbols $V$, including a special start symbol $S^{\dagger}$; a set of terminal symbols $T$; and a set of rule productions $P$ of the form $A \rightarrow \alpha$ for $A \in V$ and $\alpha \in(V \cup T)^{*}$, i.e., a single non-terminal on the left-hand side of the rule production, and a sequence of 0 or more terminals or non-terminals on the right-hand side of the rule. If we have a rule production $A \rightarrow B C$ in $P$, a completed $B$ entry in chart cell $(i, j)$ and a completed $C$ entry in chart cell $(j, k)$, then we can place a completed $A$ entry in chart cell $(i, k)$, typically with some indication that the $A$ was built from the $B$ and $C$ entries. Such a chart cell entry is sometimes called an "edge".

The issue with incomplete edges arises when there are rule productions in $P$ with more than two children on the right-hand side of the rule. Rather than trying to combine an arbitrarily large number of smaller cell entries, a more efficient approach, which exploits shared structure between rules, is to only perform pairwise combination, and store incomplete edges to represent combinations that require further combination to achieve a complete edge. This can either be performed in advance, e.g., by factoring a grammar to be in Chomsky Normal Form, as required by the CYK algorithm (Cocke and Schwartz, 1970; Younger, 1967; Kasami, 1965), resulting in "incomplete" non-terminals created by the factorization; or incomplete edges can be represented through socalled dotted rules, as with the Earley (1970) algorithm, in which factorization is essentially performed on the fly. For example, if we have a rule production $A \rightarrow B C D$ in $P$, a completed $B$ entry in chart cell $(i, j)$ and a completed $C$ entry in chart cell $(j, k)$, then we can place an incomplete edge $A \rightarrow B C \cdot D$ in chart cell $(i, k)$. The dot signifies the division between what has already been combined (to the left of the dot), and what remains to be combined. ${ }^{1}$ Then, if we have an incomplete edge $A \rightarrow B C \cdot D$ in chart cell $(i, k)$ and a complete $D$ in cell $(k, l)$, we can place a completed $A$ entry in chart cell $(i, l)$.

If a chart cell $(i, j)$ has been "closed" due to constraints limiting multi-word constituents with that span - either $w_{i} \in S_{1}$ or $w_{j} \in E_{1}($ and $i<j$ ) then it is clear that "complete" edges should not be entered in the cell, since these represent precisely the multi-word constituents that are being ruled out. How about incomplete edges? To the extent that an incomplete edge can be extended to a valid complete edge, it should be allowed. There are two cases. If $w_{i} \in S_{1}$, then under the assumption that incomplete edges are extended from left-toright (see footnote 1), the incomplete edge should

\footnotetext{
${ }^{1}$ Without loss of generality, we will assume that edges are extended from left-to-right.
} 


\begin{tabular}{|l|c|c|c|c|}
\hline \multirow{2}{*}{ Parsing constraints } & \multicolumn{3}{|c|}{ Parsing accuracy } & \% of Cells \\
\cline { 2 - 4 } & LR & LP & F & Closed \\
\hline None (baseline) & 88.6 & 89.2 & 88.9 & - \\
\hline$S_{1}$ positions & 87.6 & 89.1 & 88.3 & 44.6 \\
\hline$E_{1}$ positions & 87.4 & 88.5 & 87.9 & 66.4 \\
\hline Both $S_{1}$ and $E_{1}$ & 86.5 & 88.6 & 87.4 & 80.3 \\
\hline
\end{tabular}

Table 3: Charniak parsing accuracy on section 24 under various constraint conditions, using word labels extracted using Markov order 1 model.

be discarded, because any completed edges that could result from extending that incomplete edge would have the same start position, i.e., the chart cell would be $(i, k)$ for some $k>i$, which is closed to the completed edge. However, if $w_{i} \notin S_{1}$, then $w_{j} \in E_{1}$. A complete edge achieved by extending the incomplete edge will end at $w_{k}$ for $k>j$, and cell $(i, k)$ may be open, hence the incomplete edge should be allowed in cell $(i, j)$. See $\S 6$ for limitations on how such incomplete edges arise in closed cells, which has consequences for the worst-case complexity under certain conditions.

\section{Constraining the Charniak parser}

\subsection{Parser overview and constraint methods}

The Charniak (2000) parser is a multi-stage, agenda-driven, edge-based parser, that can be constrained by precluding edges from being placed on the agenda. Here we will briefly describe the overall architecture of that parser, and our method for constraining its search.

The first stage of the Charniak parser uses an agenda and a simple PCFG to build a sparse chart, which is used in later stages with the full model. We will focus on this first stage, since it is here that we will be constraining the parser. The edges on the agenda and in the chart are dotted rules, as described in $\S 4$. When edges are created, they are pushed onto the agenda. Edges that are popped from the agenda are placed in the chart, and then combined with other chart entries to create new edges that are pushed onto the agenda. When a complete edge spanning the whole string is placed in the chart, at least one full solution exists in the chart. After this happens, the parser continues adding edges to the chart and agenda until reaching some parameterized target number of additional edges in the chart, at which point the next stage of the pipeline receives the chart as input and any edges remaining on the agenda are discarded.

We constrain the first stage of the Charniak parser as follows. Using classifiers, a subset of word positions are assigned to class $S_{1}$, and a subset are assigned to class $E_{1}$. (Words can be assigned to both.) When an edge is created for cell $(i, j)$, where $i<j$, it is not placed on the agenda if either of the following two conditions hold: 1) $w_{i} \in S_{1}$; or 2) the edge is complete and $w_{j} \in E_{1}$.

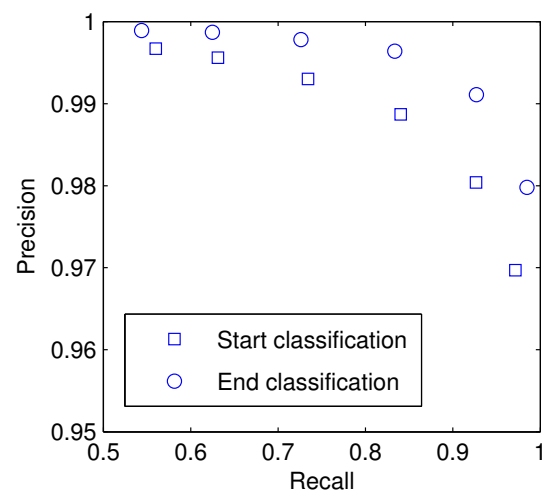

Figure 2: Precision/recall tradeoff of $S_{1}$ and $E_{1}$ tags on the development set.

Of course, the output of our classifier is not perfect, hence imposing these constraints will sometimes rule out the true parse, and parser accuracy may degrade. Furthermore, because of the agendabased heuristic search, the efficiency of search may not be impacted as straightforwardly as one might expect for an exact inference algorithm. For these reasons, we have performed extensive empirical trials under a variety of conditions to try to clearly understand the best practices for using these sorts of constraints for this sort of parser.

\subsection{Experimental trials}

We begin by simply taking the output of the Markov order 1 taggers, whose accuracies are reported in Table 2, and using word positions labeled as $S_{1}$ or $E_{1}$ to "close" cells in the Charniak parser, as described above. Table 3 presents parser accuracy on the development set (section 24) under four conditions: the unconstrained baseline; using just $S_{1}$ words to close cells; using just $E_{1}$ word positions to close cells; and using both $S_{1}$ and $E_{1}$ positions to close cells. As can be seen from these results, all of these trials result in a decrease in accuracy from the baseline, with larger decreases associated with higher percentages of closed cells.

These results indicate that, despite the relatively high accuracy of classification, the precision of our classifier in producing the $S_{1}$ and $E_{1}$ tags is too low. To remedy this, we traded some recall for precision as follows. We used the forward-backward algorithm with our Markov order 1 tagging model to assign a conditional probability at each word position of the tags $S_{1}$ and $E_{1}$ given the string. At each word position $w_{i}$ for $1<i<n$, we took the $\log$ likelihood ratio of tag $S_{1}$ as follows:

$$
\operatorname{LLR}\left(w_{i} \in S_{1}\right) \quad=\quad \log \frac{\mathrm{P}\left(w_{i} \in S_{1} \mid w_{1} \ldots w_{n}\right)}{\mathrm{P}\left(w_{i} \notin S_{1} \mid w_{1} \ldots w_{n}\right)}
$$

and the same for tag $E_{1}$. A default classification threshold is to label $S_{1}$ or $E_{1}$ if the above log likelihood is greater than zero, i.e., if the $S_{1}$ tag is more likely than not. To improve the precision, we can move this threshold to some greater value. 


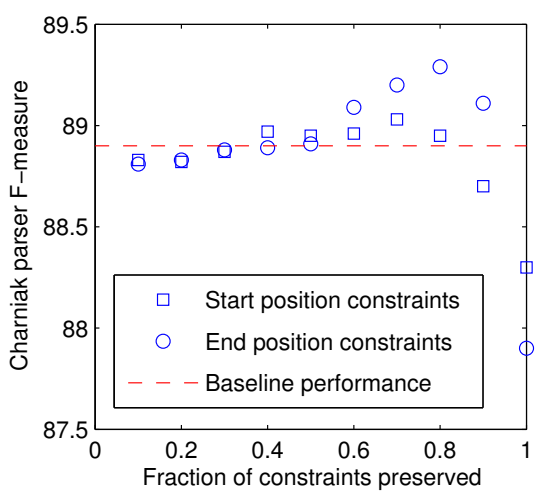

Figure 3: Charniak parser F-measure at various operating points of the fraction $c$ of total constraints kept.

Each word position in a string was ranked with respect to these log likelihood ratios for each tag. ${ }^{2}$ If the total number of words $w_{i}$ with $\operatorname{LLR}\left(w_{i} \in S_{1}\right)>0$ is $k$, then we defined multiple operating points by setting the threshold such that $c k$ words remained above threshold, for some constant $c$ between 0 and 1. Fig. 2 shows the precision/recall tradeoff at these operating points for both $S_{1}$ and $E_{1}$ tags. Note that for both tags, we can achieve over $99 \%$ precision with recall above $70 \%$, and for the $E_{1} \operatorname{tag}$ (a more frequent class than $S_{1}$ ) that level of precision is achieved with recall greater than $90 \%$.

Constraints were derived at each of these operating points and used within the Charniak parsing pipeline. Fig. 3 shows the F-measure parsing performance using either $S_{1}$ or $E_{1}$ constraints at various values of $c$ for preserving $c k$ of the original $k$ constraints. As can be seen from that graph, with improved precision both types of constraints have operating points that achieve accuracy improvements over the baseline parser on the dev set under default parser settings.

This accuracy improvement is similar to results obtained in Hollingshead and Roark (2007), where base phrase constraints from a finite-state chunker were used to achieve improved parse accuracy. Their explanation for the accuracy improvement, which seems to apply in this case as well, is that the first stage of the Charniak parser is still passing the same number of edges in the chart to the second stage, but that the edges now come from more promising parts of the search space, i.e., the parser does a better job of exploring good parts of the search space. Hence the constraints seem to be doing what they should do, which is constrain the search without unduly excluding good solutions.

Note that these results are all achieved with the default parsing parameterizations, so that accuracy gains are achieved, but not necessarily efficiency gains. The Charniak parser allows for

\footnotetext{
${ }^{2}$ Perceptron weights were interpreted in the log domain and conditionally normalized appropriately.
}

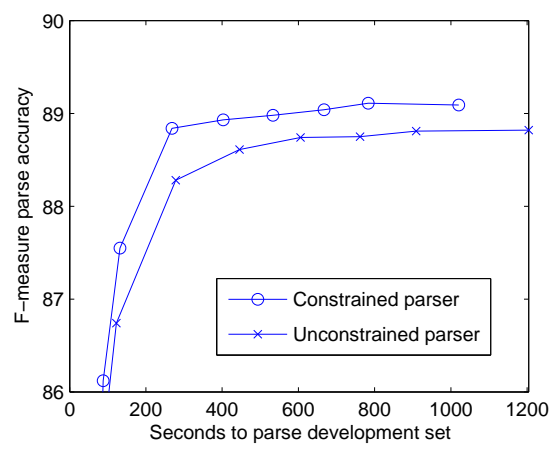

Figure 4: Speed/accuracy tradeoff for both the unconstrained Charniak parser and when constrained with high precision start/end constraints.

narrow search parameterizations, whereby fewer edges are added to the chart in the initial stage. Given the improved search using these constraints, high accuracy may be achieved at far narrower search parameterizations than the default setting of the parser. To look at potential efficiency gains to be had from these constraints, we chose the most constrained operating points for both start and end constraints that do not hurt accuracy relative to the baseline parser ( $c=0.7$ for $S_{1}$ and $c=0.8$ for $E_{1}$ ) and used both kinds of constraints in the parser. We then ran the Charniak parser with varying search parameters, to observe performance when search is narrower than the default. Fig. 4 presents F-measure accuracy for both constrained and unconstrained parser configurations at various search parameterizations. The times for the constrained parser configurations include the approximately 20 seconds required for POS-tagging and word-boundary classification of the dev set.

These results demonstrate a sharper knee of the curve for the constrained runs, with parser accuracy that is above that achieved by the unconstrained parser under the default search parameterization, even after a nearly 5 times speedup.

\subsection{Analysis of constraints on 1-best parses}

There are two ways in which the constraints could be improving parser performance: by helping the parser to find higher probability parses that it was formerly losing because of search errors; or by not allowing the parser to select high probability parses that violate the constraints. To get a sense of whether the constraints on the parser are simply fixing search errors or are imposing constraints on the model itself, we examined the 1-best parses from both constrained and unconstrained scenarios. First, we calculated the geometric mean of the 1-best parse probabilities under both scenarios, which were (in logs) -207.99 for unconstrained and -208.09 for constrained. Thus, the constrained 1-best parses had very slightly less probability than the unconstrained parses, indicating that the constraints were not simply fixing search er- 
rors, but also eliminated some MAP parses.

To get a sense of how often search errors were corrected versus ruling out of MAP parses, we compared the constrained and unconstrained parses at each string, and tallied when the unconstrained parse probabilities were greater (or less) than the constrained parse probabilities, as well as when they were equal. At the default search parameterization (210), 84.8 percent of the strings had the same parses; in 9.2 percent of the cases the unconstrained parses had higher probability; and in 5.9 percent of the cases the constrained parses had higher probability. The narrower search parameterization at the knee of the curve in Fig. 4 had similar results: 84.6 percent were the same; in 8.6 percent of the cases the unconstrained probability was higher; and in 6.8 percent of the cases the constrained probability was higher. Hence, when the 1-best parse differs, the parse found via constraints has a higher probability in approximately 40 percent of the cases.

\section{$6 O\left(n^{2}\right)$ complexity context-free parsing}

Using sufficient $S_{1}$ and $E_{1}$ constraints of the sort we have been investigating, we can achieve worstcase quadratic (instead of cubic) complexity. A proof, based on the CYK algorithm, is given in Appendix A, but we can make the key points here. First, cubic complexity of context-free inference is due to $O\left(n^{2}\right)$ chart cells and $O(n)$ possible child configurations per cell. If we "close" all but $O(n)$ cells, the "open" cells will be processed with worst-case quadratic complexity $(O(n)$ cells with $O(n)$ possible child configurations per cell). If we can show that the remaining $O\left(n^{2}\right)$ "closed" cells each can be processed within constant time, then the overall complexity is quadratic. The proof in Appendix A shows that this is the case if closing a cell is such that: when a cell $(i, j)$ is closed, then either all cells $(i, k)$ for $k>i$ are closed or all cells $(k, j)$ for $k<j$ are closed. These conditions are achieved when we select sets $S_{1}$ and $E_{1}$ and close cells accordingly.

Just as we were able to order word position log likelihood scores for classes $S_{1}$ and $E_{1}$ to improve precision in the previous section, here we will order them so that we can continue selecting positions until we have guaranteed less than some threshold of "open" cells. If the threshold is linear in the length of the string, we will be able to parse the string with worst-case quadratic complexity, as shown in Appendix A. We will set our threshold to $k n$ for some constant $k$ (in our experiments, $k$ ranges from 2 to 10 ). Table 4 presents the percentage of cells closed, class $\left(S_{1}\right.$ and $\left.E_{1}\right)$ precision and parser accuracy when the number of "open" cells is bounded to be less than

\begin{tabular}{|c|c|c|c|c|c|}
\hline Open & \% cells & Class & \multicolumn{3}{|c|}{ Parse accuracy } \\
cells & closed & Prec & LR & LP & F \\
\hline all & - & - & 88.6 & 89.2 & 88.9 \\
\hline $10 n$ & 39.1 & 99.9 & 88.6 & 89.2 & 88.9 \\
\hline $8 n$ & 50.4 & 99.9 & 88.6 & 89.2 & 88.9 \\
\hline $6 n$ & 62.8 & 99.9 & 88.6 & 89.2 & 88.9 \\
\hline $4 n$ & 75.7 & 99.8 & 88.8 & 89.4 & 89.1 \\
\hline $2 n$ & 88.8 & 99.8 & 88.8 & 89.5 & 89.1 \\
\hline
\end{tabular}

Table 4: Varying constant $k$ for $k n$ "open" cells, yielding $\mathrm{O}\left(n^{2}\right)$ parsing complexity guarantees

the threshold. These results clearly demonstrate that such constraints can be placed on real contextfree parsing problems without significant impact to accuracy-in fact, with small improvements.

We were quite surprised by these trials, fully expecting these limits to negatively impact accuracy. The likely explanation is that the existing Charniak search strategy itself is bounding processing in such a way that the additional constraints placed on the process do not interfere with standard processing. Note that our approach closes a higher percentage of cells in longer strings, which the Charniak pipeline already more severely prunes than shorter strings. Further, this approach appears to be relying very heavily on $E_{1}$ constraints, hence has very high precision of classification.

While the Charniak parser may not be the ideal framework within which to illustrate these worstcase complexity improvements, the lack of impairment to the parser provides strong evidence that other parsers could make use of the resulting charts to achieve significant efficiency gains.

\section{Conclusion \& Future Work}

In this paper, we have presented a very simple approach to constraining context-free chart parsing pipelines that has several nice properties. First, it is based on a simple classification task that can achieve very high accuracy using very simple models. Second, the classifier output can be straightforwardly used to constrain any chartbased context-free parser. Finally, we have shown (in Appendix A) that "closing" sufficient cells with these techniques leads to quadratic worst-case complexity bounds. Our empirical results with the Charniak parser demonstrated that our classifiers were sufficiently accurate to allow for such bounds to be placed on the parser without hurting parsing accuracy.

Future work in this direction will involve trying different methods for defining effective operating points, such as more heavily constraining longer strings, in an attempt to further improve the search in the Charniak parser. We would also like to investigate performance when using other chart parsing strategies, such as when using cell pruning instead of an agenda. 


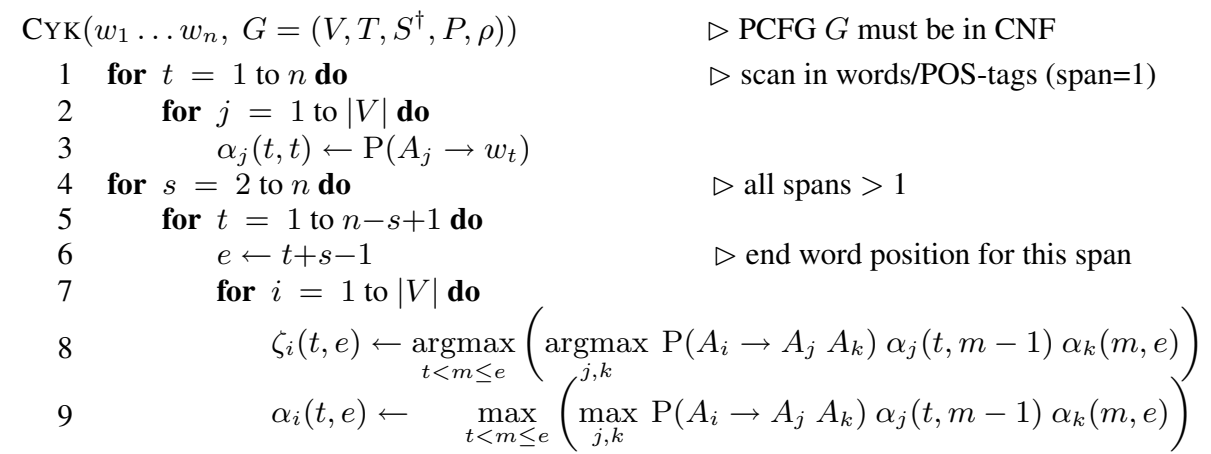

Figure 5: Pseudocode of a basic CYK algorithm for PCFG in Chomsky Normal Form (CNF).

\section{References}

Charniak, E. and M. Johnson. 2005. Coarse-to-fine $n$-best parsing and MaxEnt discriminative reranking. In Proceedings of the 43rd Annual Meeting of the Association for Computational Linguistics (ACL), pages 173-180.

Charniak, E. 2000. A maximum-entropy-inspired parser. In Proceedings of the 1st Conference of the North American Chapter of the Association for Computational Linguistics, pages 132-139.

Cocke, J. and J.T. Schwartz. 1970. Programming languages and their compilers: Preliminary notes. Technical report, Courant Institute of Mathematical Sciences, NYU.

Collins, M.J. 2002. Discriminative training methods for hidden Markov models: Theory and experiments with perceptron algorithms. In Proceedings of the Conference on Empirical Methods in Natural Language Processing (EMNLP), pages 1-8.

Earley, J. 1970. An efficient context-free parsing algorithm. Communications of the ACM, 6(8):451-455.

Glaysher, E. and D. Moldovan. 2006. Speeding up full syntactic parsing by leveraging partial parsing decisions. In Proceedings of the COLING/ACL 2006 Main Conference Poster Sessions, pages 295-300.

Hollingshead, K. and B. Roark. 2007. Pipeline iteration. In Proceedings of the 45th Annual Meeting of the Association for Computational Linguistics (ACL), pages 952-959.

Kasami, T. 1965. An efficient recognition and syntax analysis algorithm for context-free languages. Technical report, AFCRL-65-758, Air Force Cambridge Research Lab., Bedford, MA.

Marcus, M.P., M.A. Marcinkiewicz, and B. Santorini. 1993. Building a large annotated corpus of English: The Penn treebank. Computational Linguistics, 19:313-330.

Ratnaparkhi, A. 1999. Learning to parse natural language with maximum entropy models. Machine Learning, 34(13):151-175

Sha, F. and F. Pereira. 2003. Shallow parsing with conditional random fields. In Proceedings of HLT-NAACL, pages 134-141.

Younger, D.H. 1967. Recognition and parsing of contextfree languages in time $\mathrm{n}^{3}$. Information and Control, 10(2):189-208.

\section{Appendix A Proof of quadratic complexity parsing with constraints}

For this proof, we will use the well-known CYK parsing algorithm, which makes use of grammars in Chomsky Normal Form (CNF). To achieve $\mathrm{CNF}$, among other things, rules with more than 2 children on the right-hand side must be factored into multiple binary rules. To do this, composite non-terminals are created in the factorizations, which represent incomplete constituents, i.e., those edges that require further combination to be made complete. ${ }^{3}$ For example, if we have a rule production $A \rightarrow B C D$ in the context-free grammar $G$, then a new composite non-terminal would be created, e.g., $B-C$, and two binary rules would replace the previous ternary rule: $A \rightarrow B-C D$ and $B-C \rightarrow B C$. The $B-C$ non-terminal represents part of a rule expansion that needs to be combined with something else to produce a complete nonterminal from the original set of non-terminals. Let $V^{\prime}$ be the set of non-terminals that are created through factorization, which hence represent incomplete edges.

Fig. 5 shows pseudocode of a basic CYK algorithm for use with a probabilistic $\mathrm{CFG}$ in $\mathrm{CNF}$, $G=\left(V, T, S^{\dagger}, P, \rho\right)$. The function $\rho$ maps from rules in $P$ to probabilities. Lines $1-3$ of the algorithm in Fig. 5 initialize the span 1 cells. Lines 4-9 are where the cubic complexity comes in: $O(n)$ loops in line 4, each of which include $O(n)$ loops in line 5, each of which requires finding a maximum over $O(n)$ midpoints $m$ in lines 8-9. For each non-terminal $A_{i} \in V$ at each cell $(t, e)$, the algorithm stores a backpointer $\zeta_{i}(t, e)$ in line 8 , for efficiently extracting the maximum likelihood solution at the end of inference; and maximum probabilities $\alpha_{i}(t, e)$ in line 9 , for use in the dynamic program.

Given a set of word positions in the classes $S_{1}$ and $E_{1}$, as defined in the main part of this paper, we can designate all cells $(i, j)$ in the chart where either $w_{i} \in S_{1}$ or $w_{j} \in E_{1}$ to be "closed". Chart cells that are not closed will be called "open". The total number of cells in the chart is $\left(n^{2}+n\right) / 2$, and if we set a threshold on the maximum number of open cells to be $k n$, the number of closed cells must be at least $\left(n^{2}+n\right) / 2-k n$. Given an ordering of words (see $\S 6$ for one approach), we can add words to these sets one word at a time and close the

\footnotetext{
${ }^{3}$ As before, we assume that edges are extended from leftto-right, which requires a left-factorization of the grammar.
} 


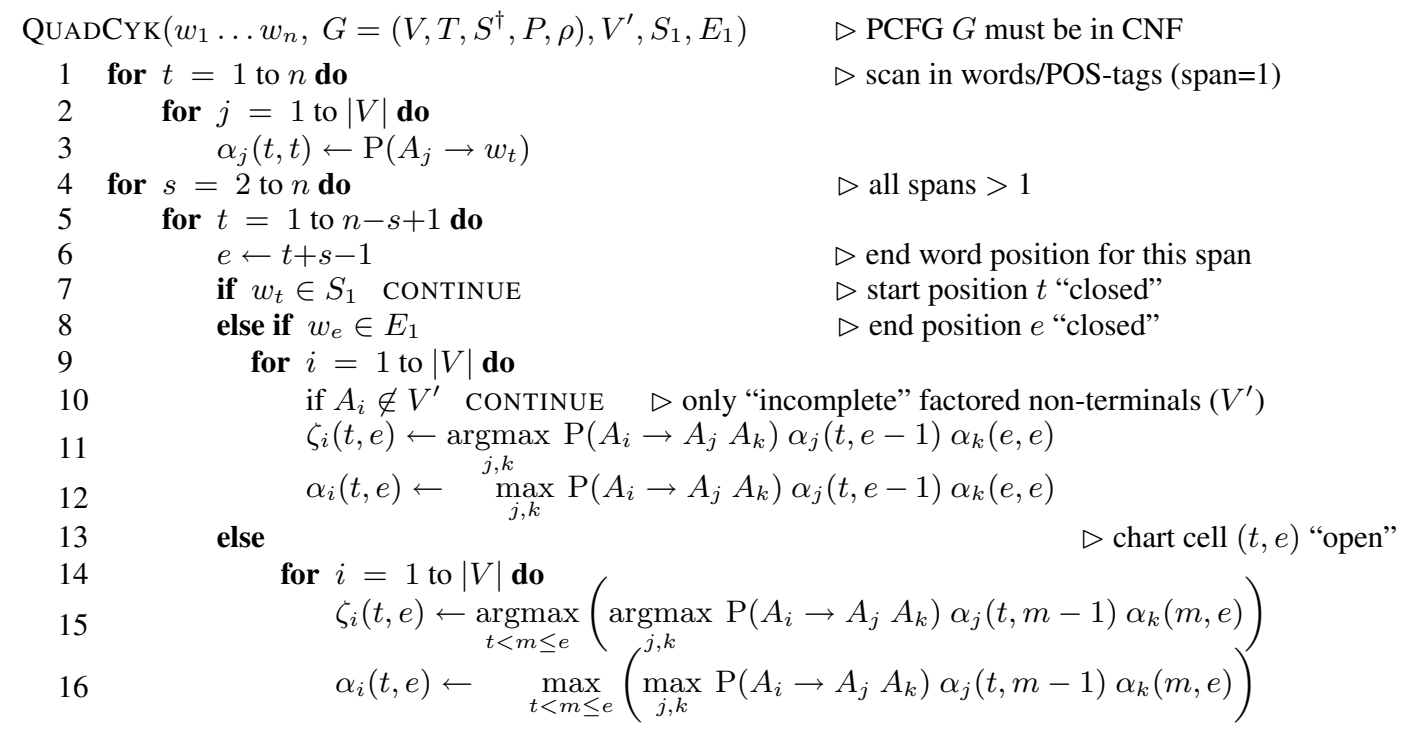

Figure 6: Pseudocode of a modified CYK algorithm, with quadratic worst case complexity with $O(n)$ "open" cells. In addition to string and grammar, it requires specification of factored non-terminal set $V^{\prime}$ and position constraints $\left(S_{1}, E_{1}\right)$.

related cells, until the requisite number of closures are achieved. Then the resulting sets of $S_{1}$ word positions and $E_{1}$ word positions can be provided to the parsing algorithm, in addition to the grammar $G$ and the set of factored non-terminals $V^{\prime}$.

Fig. 6 shows pseudocode of a modified CYK algorithm that takes into account $S_{1}$ and $E_{1}$ word classes. Lines 1-6 of the algorithm in Fig. 6 are identical to those in the algorithm in Fig. 5. At line 7 , we have identified the chart cell being processed, which is $(t, e)$. If $w_{t} \in S_{1}$ then the cell is completely closed, and there is nothing to do. Otherwise, if $w_{e} \in E_{1}$ (lines 8-12), then factored nonterminals from $V^{\prime}$ can be created in that cell by finding legal combinations of children categories. If neither of these conditions hold, then the cell is open (lines 13-16) and processing occurs as in the standard CYK algorithm (lines 14-16 of the algorithm in Fig. 6 are identical to lines 7-9 in Fig. 5).

If the number of "open" cells is less than $k n$ for some constant $k$, then we can prove that the algorithm in Fig. 6 is $O\left(n^{2}\right)$ when given a left-factored grammar in CNF. A key part of the proof rests on two lemmas:

Lemma 1: Let $V^{\prime}$ be the set of composite nonterminals created when left-factoring a CFG to be in CNF, as described earlier. Then, for any production $A_{i} \rightarrow A_{j} A_{k}$ in the grammar, $A_{k} \notin V^{\prime}$.

Proof: With left-factoring, any k-ary production $A \rightarrow A_{1} \ldots A_{k-1} A_{k}$ results in new non-terminals that concatenate the first $k-1$ non-terminals on the right-hand side. These factored non-terminals are always the leftmost child in the new production, hence no second child in the resulting CNF grammar can be a factored non-terminal.

Lemma 2: For a cell $(t, e)$ in the chart, if $w_{e} \in E_{1}$, then the only possible midpoint $m$ for creating an entry in the cell is $e$.

Proof: Placing an entry in cell $(t, e)$ requires a rule $A_{i} \rightarrow A_{j} A_{k}$, an $A_{j}$ entry in cell $(t, m-1)$ and an $A_{k}$ entry in cell $(m, e)$. Suppose there is an $A_{k}$ entry in cell $(m, e)$ for $m<e$. Recall that $w_{e} \in E_{1}$, hence the cell $(m, e)$ is closed to non-terminals not in $V^{\prime}$. By Lemma $1, A_{k} \notin V^{\prime}$, therefore the cell $(m, e)$ is closed to $A_{k}$ entries. This is a contradiction. Therefore, the lemma is proved. $\square$

Theorem: Let $\mathcal{O}$ be the set of cells $(t, e)$ such that $w_{t} \notin S_{1}$ and $w_{e} \notin E_{1}$ ("open” cells). If $|\mathcal{O}|<k n$ for some constant $k$, where $n$ is the length of the string, then the algorithm in Fig. 6 has worst case complexity $O\left(n^{2}\right)$.

Proof: Lines 4 and 5 of the algorithm in Fig. 6 loop through $O\left(n^{2}\right)$ cells $(t, e)$, for which there are three cases: $w_{t} \in S_{1}$ (line 7 of Fig. 6); $w_{e} \in E_{1}$ (lines 8-12); and $(t, e) \in \mathcal{O}$ (lines 13-16).

Case 1: $w_{t} \in S_{1}$. No further work to be done.

Case 2: $w_{e} \in E_{1}$. There is a constant amount of work to be done, for the reason that there is only one possible midpoint $m$ for binary children combinations (namely $e$, as proved in Lemma 2), hence no need to perform the maximization over $O(n)$ midpoints.

Case 3: $(t, e) \in \mathcal{O}$. As with standard CYK processing, there are $O(n)$ possible midpoints $m$ over which to maximize, hence $O(n)$ work required.

Only $O(n)$ cells fall in case 3 , hence the total amount of work associated with the cells in $\mathcal{O}$ is $O\left(n^{2}\right)$. There are $O\left(n^{2}\right)$ cells associated with cases 1 and 2 , each of which has a total amount of work bounded by a constant, hence the total amount of work associated with the cells not in $\mathcal{O}$ is also $O\left(n^{2}\right)$. Therefore the overall worst-case complexity of the algorithm under these conditions is $O\left(n^{2}\right)$. 